\title{
Superquadrics and free-form deformations : a global model to fit and track 3D medical data
}

\author{
Eric Bardinet, Laurent D. Cohen ${ }^{\star}$ \\ Nicholas Ayache \\ INRIA Sophia Antipolis \\ 2004, Route des Lucioles BP 93 \\ 06902 Sophia Antipolis CEDEX, France. \\ Email: bard@epidaure.inria.fr
}

\begin{abstract}
Recovery of $3-D$ data with simple parametric models has been the subject of many studies over the last ten years. Many have used the notion of superquadrics, introduced for graphics in [4]. It appears, however, that although superquadrics can describe a wide variety of forms, they are too simple to recover and describe complex shapes.

This paper describes a method to fit to 3-D points and then track a parametric deformable surface. We suppose that a 3-D image has been segmented to get a set of 3-D points. A first estimate consists of our version of a superquadric fit with global tapering. We then apply the technique of free-form deformations, as introduced by [9] in computer graphics to refine the estimate. We present experimental results with real $3 \cdot D$ medical images, where the original points are laid on an iso-surface. This is also applied to give efficient tracking of the deformation of the myocardium
\end{abstract}

\section{Introduction}

Over the last ten years, many surface reconstruction problems have been formulated as the minimization of an energy function corresponding to a model of the surface. Using deformable models and templates, the extraction of a shape is obtained through an energy composed of an internal regularization term and an external attraction potential (data term), illustrated for example in [13, 6, 10, 12]. Since the relevant surfaces in medical images are usually smooth, the use of such models is often very efficient for locating surface boundaries of organs and structures, and for the subsequant tracking of these shapes in a time sequence.

The advantage of deformable templates like superquadrics is their small number of parameters to represent a shape. However, if superquadric shapes give a good global approximation to a surface, the set of shapes described by superquadrics is too limited to approximate precisely complex surfaces. Therefore they were coupled with a deformable model in [12] to take into account local deformations.

* CEREMADE, U.R.A. CNRS 749, Université Paris IX- Dauphine, Place du Marechal de Lattre de Tassigny 75775 Paris CEDEX 16, France 
The contribution of this work is twofold. First, we propose an algorithm for fitting data with a superquadric, based on [10] with some variations. Second, we improve the shape extraction by introducing the use of free-form deformations (FFD), as introduced by [9] in computer graphics. The idea is to put our previous surface, here a superquadric, in a rubber-like box and then to deform this box by moving its control points. We solve an inverse problem to find the set of control points which minimize the error in the displacement field on the whole object. FFD has also been successfully used by [11] to match anatomical 3-D surfaces.

We show example results for 3-D medical images of the myocardium where the data set is an iso-surface. We also show an efficient tracking of the deformation of the myocardium.

\section{Superquadric Fitting}

This class of objects was introduced to computer graphics by A. Barr ([4]) and is an extension to $3-\mathrm{D}$ of the superellipse. Their first use in computer graphics and in vision is due to Pentland ([8]), followed by Bajcsy ([10]) and later by Terzopoulos and Metaxas ( [12]). A more complete description of Superquadrics and their use in Surface Reconstruction can be found in [3].

\subsection{Definition of Superquadrics}

Superquadrics form a family of implicit surfaces obtained by extension of the familiar set of quadrics. They are obtained by spherical product (see [4]) of two 2-D curves. The superellipsoid is the spherical product of the superellipse with itself. For a complete definition, see [2].

\subsection{Our Superquadric Fitting}

In our applications, the original data is a 3-D medical image which represents, for example, the myocardium or the head area. Interesting features can either be edges extracted from the data, corresponding to a potential (gradient), or an iso-surface. We want to approximate this surface by a superellipsoid.

To fit a superquadric to a set of data points, we presented in [2] a revised version of Solina and Bajcsy's Model of a superquadric fit. After initialization of the surface by an ellipsoid defined by the moments of inertia of the data, they made least squares minimization the inside-outside function $F$ for:

$$
E(A)=\sum_{i=1}^{N}\left[\left(1-F\left(a_{i}, \epsilon_{j}, \varphi, \theta, \psi, t\right)\right)\right]^{2} .
$$

We improved this approach by modifying the initialization, introducing global tapering and using Conjugate Gradient Descent.

\section{Free-Form Deformations}

The previous fit gives a first approximation to the surface, but it is not sufficiently close to the data. The superquadric is correctly oriented and the three axes of inertia already have the right size. The problem is that the set of shapes described 
by superquadrics is too limited to describe complex medical objects, in particular the myocardium or brain. We conclude that we need a more complex model.

In [1], we applied to surface fitting a tool called Free-Form Deformations (FFD) developed in computer graphics (see [9, 7]). This is a 3D-space deformation and consists of including a surface in a box and deforming it as a $3 \mathrm{D}$ solid. Our choice was guided by the fact that we wanted to have a simple global model at the end of the process.

\subsection{Definition}

FFD deforms solid geometric models in a free-form manner. It is independent of the nature of the object to be deformed. An analogy is to consider a rubber box in which the object is situated. Control points are placed on a regular 3D grid in the box. To deform the object, control points are moved, and the object follows accordingly. In graphics and CADs, FFD are used to design complex shapes by successively moving control points of the box to some place and thus retrieving a global deformation of the object.

The forward algorithm used for graphics is based on trivariate Bernstein polynomials, and divided into two steps:

1. Computation of the local coordinates of the object points in the frame defined by the set of control points.

2. Displacement of the control points and estimation of the new position of the object.

See [1] for more details.

\subsection{The Inverse Problem : A two-step Iterative Algorithm}

To improve the precision of the initial superquadric fit to the data, the superquadric is embedded in a parallelepiped box and a displacement field between the data and the model points is computed.

The FFD algorithm presented in the previous section can be summarised up like this: displacement of the control points permits the computation of a displacement map for any point. Here, we deal with the inverse problem. What we first determine is a displacement field on points of our surface. This displacement field joins a point of the model to the closest data point. The problem is then to find the displacement of the control points which minimizes the error between the displacement field produced by the FFD and the given one.

First Step: Computation of the displacement field. We need to associate with each point on the superquadric a data point $M$. The distance map is computed using a KD-tree algorithm (see [14] for example). As explained in [2], instead of taking the closest point on the data, we make it the other way:

- First the distance map to the superellipsoid is computed. We thus find for each data point the closest point on the superellipsoid.

- Since some points on the superellipsoid have not been reached, a displacement value is computed by interpolation. 
Second Step : Displacement of the control points. We want to find the new position of the control points of our 3D box that best recovers the displacement field obtained in the previous step. For more details, see [1].

Iterative Algorithm: Since after the control points have moved, we have a new position on the surface, the displacement field also changes, and we need to iterate these two steps to improve the quality of the approximation. This makes our algorithm somewhat resemble to the two-steps formulation of the B-splines snakes using auxiliary variables (see [5]), but a difference here is that we have a 3D deformation using 3D Bernstein trivariate polynomials for our 2D surface embedded in the $3 \mathrm{D}$ box. We now give a description of the algorithm:

- We begin with $P_{0}$ as the regularly spaced control point box; $X_{0}=B P_{0}$ represents the set of points on the parameterization of the initial superellipsoid.

- We then iterate the following steps:

Step 1: Displacement Field Computation: $X_{n}^{a}=X_{n}+\delta X_{n}$

Step 2: Control Points $P_{n+1}$ Computation by Minimization of $\left\|B P-X_{n}^{a}\right\|^{2}$

$X_{n+1}=B P_{n+1}$

Test: computation of the least-square error $\left\|X_{n+1}-X_{n}\right\|$

\subsection{Including Regularization in the Inverse Problem}

As shown in figure 1, the control points box may be very irregular. In consequence, it is unstable to study the evolution of these control points in a sequence of images. In [11], the authors deal with a similar problem and introduce $\mathrm{m}$-th order stabilizers. In order to control explicitly the regularity of the box, we add a regularization term into the minimization of the second step. The minimization criterion now becomes:

$$
\left\|B P-X_{n}^{a}\right\|^{2}+\alpha \sum_{j=1}^{N P} \sum_{j^{\prime}}\left\|P_{j}-P_{j^{\prime}}\right\|^{2},
$$

where $j^{\prime}$ corresponds to the neighbours of $\boldsymbol{P}$. The second term is an internal energy corresponding to the insertion of zero-length springs between control points. This has a regularizing effect on the box to an extent controlled by the weight $\alpha$. This regularization term can be also written $\|D P\|^{2}$ where $D$ is a matrix which represents a discretized derivative of the control points position.

We show results of this regularizing effect on the box in figure 2 to compare to the previous figure 1 . One drawback of the regularization is that the accuracy of the approximation decreases when the regularizing effect increases (see table 1).

\section{Experimental Results}

We present some results obtained from applying the two-step algorithm to isosurfaces extracted from medical data, followed by tracking results. 


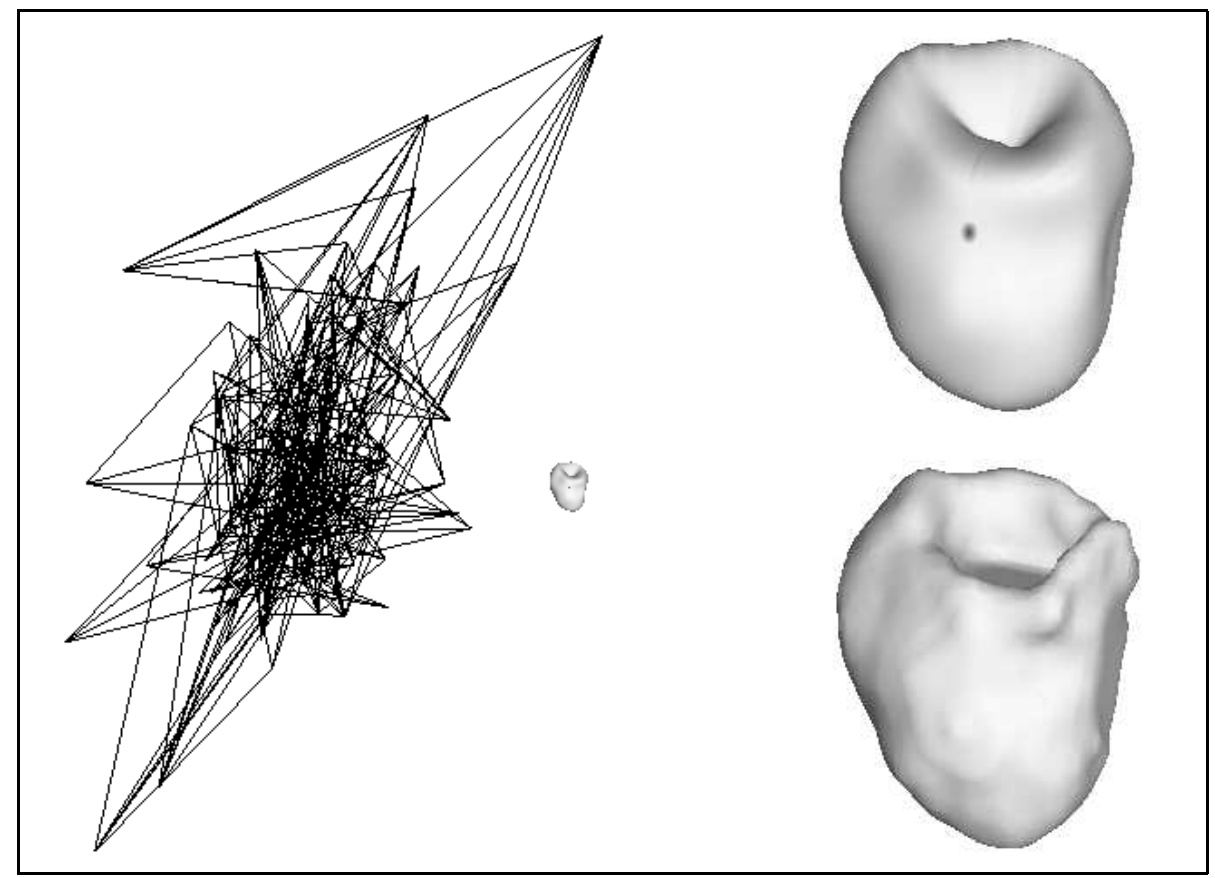

Fig. 1. Left: chaotic 5x5x5 box and the corresponding model. Right: the model compared to the original data.

\begin{tabular}{||c||c||}
\hline Regularization weight & Least-square Error \\
\hline 0.0001 & 0.015065 \\
\hline 0.001 & 0.015935 \\
\hline 0.01 & 0.017489 \\
\hline 0.1 & 0.021123 \\
\hline
\end{tabular}

Table 1. Least-square Error between original data and parametric model as a function of regularization weight.

\subsection{Medical Data: Left Ventricle of the Myocardium}

In the following examples, the left ventricle of a myocardium was extracted from a time sequence of 3 D SPECT images. Figure 3 was obtained with a $5 \times 5 \times 5$ box, the iterated algorithm and resolution using Singular Value Decomposition. These data are each composed of 6000 points, and the model is defined each time by 11 parameters for the superellipsoid and $5 \times 5 \times 53 \mathrm{D}$ points for the control points box, that is less than $1303 \mathrm{D}$ points. The information is reduced by a factor of 48 . 


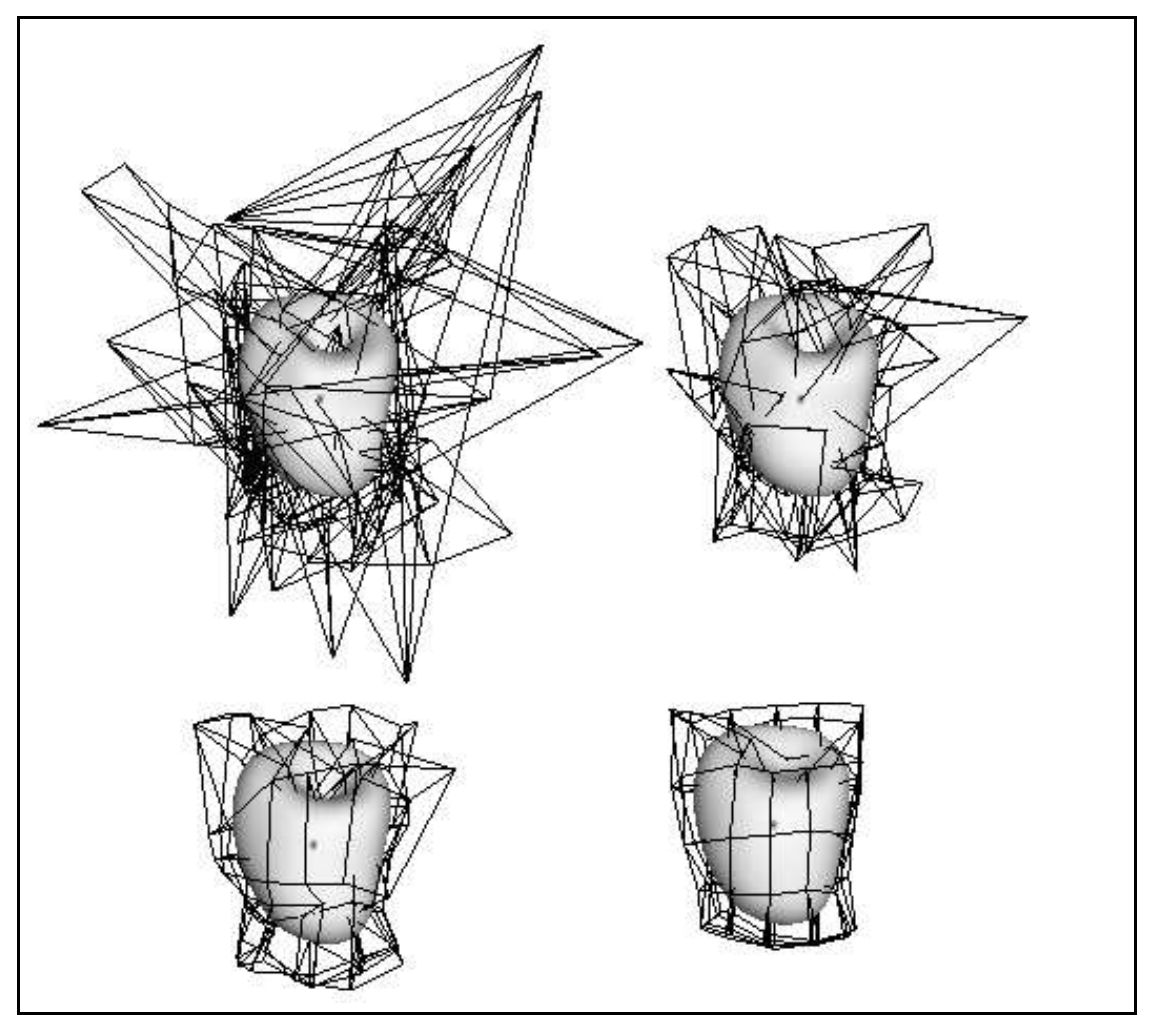

Fig. 2. Regularizing effect. Top left: $\alpha=0.0001$. Top right: $\alpha=0.001$. Bottom left: $\alpha=0.01$. Bottom right: $\alpha=0.1$.

\subsection{Tracking and detection of pathology}

Figure 4 shows the result of the algorithm on the time sequence of the left ventricle. The model at time $t_{0}$ was computed using the corresponding superellipsoid, but the models at time $t_{n}$ were computed using the previous ones (at time $\left.t_{n-1}\right)$. One basic application, having a time sequence of $3 \mathrm{D}$ data represented by parametric models, is to compute the displacement of each point on the surface during the sequence.For the left ventricle, this modelling can be efficient for the localization of pathological zones: for such zones, surface points have a small displacement, corresponding to necrosed areas on the ventricle.

\section{Conclusion}

We presented a new approach to shape reconstruction applied to 3-D medical data. It is based on a first approximation giving the best fit with a superquadric 


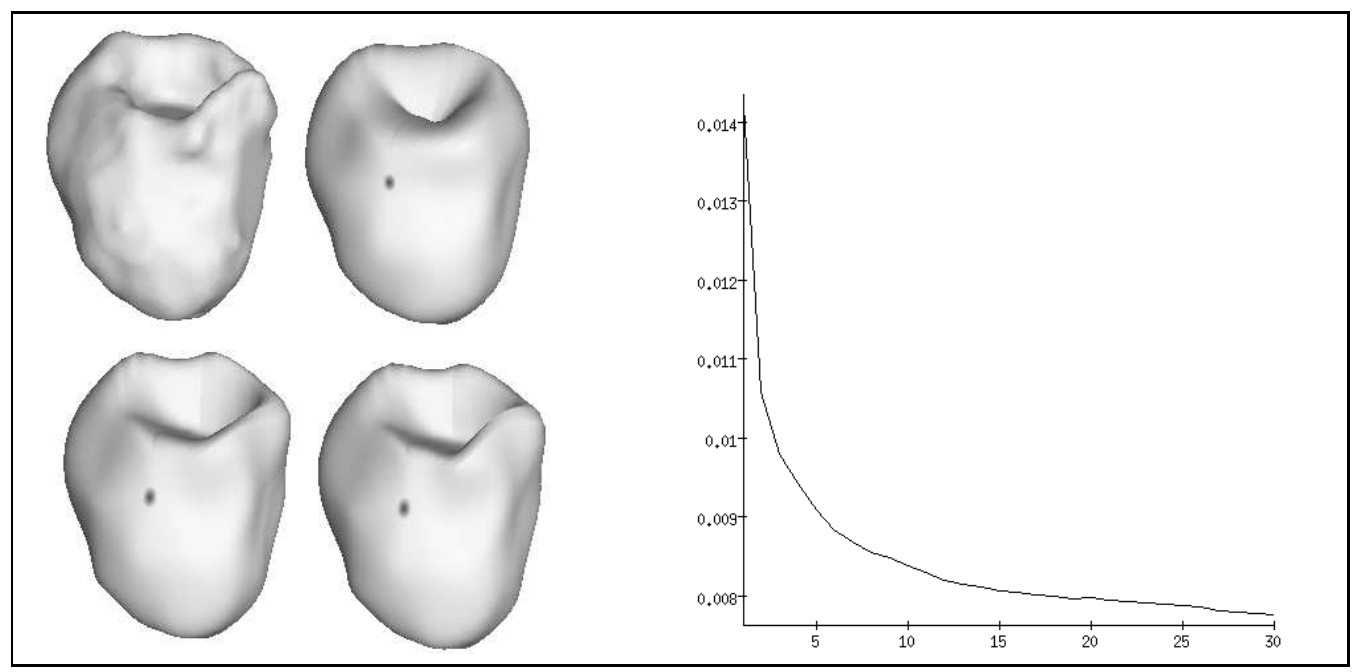

Fig. 3. Left: from top left to bottom right: left ventricle and the final result after 1, 10 and 30 iterations with a 5x5x5 box. Right: Least-square Error between original data and parametric model as a function of the number of iterations.

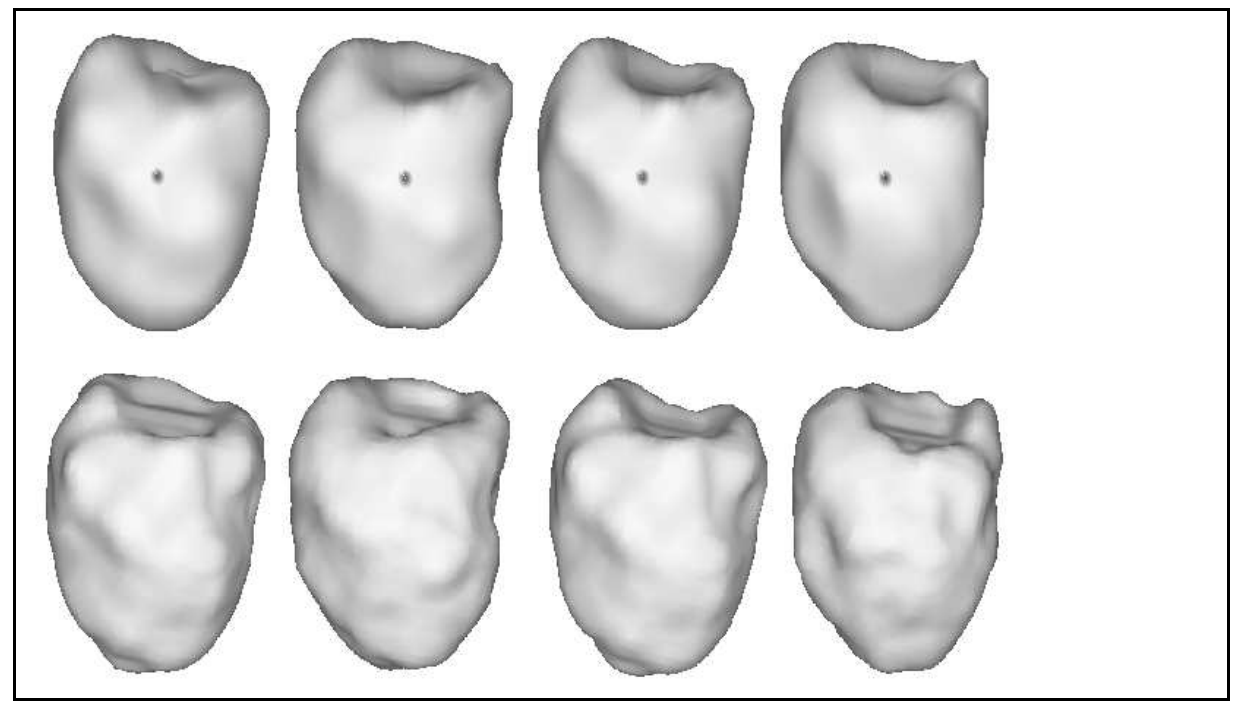

Fig. 4. Tracking the left ventricle with a 5x5x5 box. Top: the models. Bottom: the data. 
model. This is followed by a two-step algorithm for refining the details of the previous shape by making use of free-form deformations. The data is a set of points in a 3-D image, and the closest point to data is used for definition of the displacement field. We also added a regularizing term in the previous algorithm to make the control points box behave better. The interesting aspect of this approach is that it gives a description of a complex shape with only a small number of parameters.

Example results have been shown for a synthetic shape and for iso-surfaces extracted from 3-D medical images of the left ventricle. We also shown application of our method to automatic shape tracking in a time sequence of medical images.

\section{Acknowledgments}

We would like to thank Serge Benayoun and Alexis Gourdon who provided us with substantial help through fruitful discussions and Grégoire Malandain for his judicious remarks. Thanks also to Mike Brady for his careful review of this paper. This work was partially supported by Digital Equipment Corporation.

\section{References}

1. E. Bardinet, L.D. Cohen, and N. Ayache. Fitting 3-d data using superquadrics and free-form deformations. In Proceedings of the IEEE International Conference on Pattern Recognition, Jerusalem, Israel, October 1994.

2. E. Bardinet, L.D. Cohen, and N. Ayache. Fitting of iso-surfaces using superquadrics and free-form deformations. In Proceedings of the IEEE Workshop on Biomedical Images Analysis (WBIA'94), Seattle, Washington, June 1994.

3. E. Bardinet, L.D. Cohen, and N. Ayache. Representation of surfaces with a global model : Superquadrics and free-form deformations. Technical report, INRIA, October 1994. (in print).

4. A.H. Barr. Superquadrics and angle-preserving deformations. IEEE Computer Graphics Applications, 1:11-23, 1981.

5. L.D. Cohen. Use of auxiliary variables in computer vision problems. Technical Report 9409, Ceremade, February 1994. Cahiers de Mathematiques de la Decision.

6. L.D. Cohen and I. Cohen. Finite element methods for active contour models and balloons for 2-D and 3-D images. IEEE Transactions on Pattern Analysis and Machine Intelligence, 15, 1993.

7. W.M. Hsu, J.F. Hughes, and H. Kaufman. Direct manipulation of free-form deformations. In SIGGRAPH'92, volume 26, pages 177-184, Chicago, 1992.

8. A.P. Pentland. Recognition by parts. In IEEE Proceedings of the first International Conference on Computer Vision, pages 612-620, 1987.

9. T.W. Sederberg and S.R. Parry. Free-form deformation of solid geometric models. In $S I G G R A P H^{\prime} 86$, volume 20, pages 151-160, Dallas, 1986.

10. F. Solina and R. Bajcsy. Recovery of parametric models from range images : the case for superquadrics with global deformations. IEEE Transactions on Pattern Analysis and Machine Intelligence, 12:131-147, 1990.

11. R. Szeliski and S. Lavallée. Matching 3-d anatomical surfaces with non-rigid deformations using octree-splines. In Proceedings of the IEEE Workshop on Biomedical Images Analysis (WBIA'94), Seattle, Washington, June 1994. 
12. D. Terzopoulos and D. Metaxas. Dynamic 3d models with local and global deformations: deformable superquadrics. IEEE Transactions on Pattern Analysis and Machine Intelligence, 13(7):703-714, 1991.

13. D. Terzopoulos, A. Witkin, and M. Kass. Constraints on deformable models: recovering 3D shape and nonrigid motion. AI Journal, 36:91-123, 1988.

14. Z. Zhang. Iterative point matching for registration of free-form curves and surfaces. International Journal On Computer Vision, 13(2):119-152, 1994. Also Research Report No.1658, INRIA Sophia-Antipolis, 1992.

This article was processed using the $\mathrm{L} \mathrm{T}_{\mathrm{E}} \mathrm{X}$ macro package with LLNCS style 